\title{
Instrumentation Concepts for the Very Large Hadron Collider (VLHC)
}

\author{
G. William Foster \\ Fermi National Accelerator Laboratory \\ PO Box 500 Batavia IL 60510
}

\begin{abstract}
Instrumentation concepts for the Very Large Hadron Collider (VLHC) are discussed. Different design concepts for the VLHC result in substantially different instrumentation layouts. High field, cold bore magnets have instrumentation requirements very similar to the SSC and LHC. In contrast, the low field warm bore "transmission line" magnets have very sparse instrumentation and the long magnet length allows the cable plant to be preinstalled on the magnets. Specialized beam instrumentation concepts including permanently sealed semi-rigid coax BLM's and distributed coupled-bunch damping systems are discussed.
\end{abstract}

\section{THE VLHC PROJECT}

The Very Large Hadron Collider project (www.VLHC.org) is a multi-nationallaboratory collaboration initiated at the request of the Fermilab Directorate to pursue the possibility of a future U.S. machine on the energy frontier. By collaboration consensus, Fermilab will be the presumed site of the VLHC due to the excellent geological conditions and the existence of a generally functional injector and laboratory infrastructure. By necessity the machine must be a superconducting proton-proton collider, since this is the only realistic candidate for reaching the 10 $\mathrm{TeV}$ constituent center-of-mass energy scale. The reason for this unique capability comes from fundamental physics: Superconducting proton synchrotrons are based on three essentially lossless processes: i) Recirculating protons in a magnetic guide field, ii) Superconducting current transport, and iii) DC magnetization of iron blocks. Equally importantly, the beam energy is not thrown away many times a second.

The technology for building superconducting synchrotrons is mature. Magnets with appropriate properties can be built with existing materials. The beam dynamics of the machines are well understood and are not wild extrapolations from existing machines that work well. If desired, a technically defensible design could be obtained by direct scaling of either the SSC or LHC machine designs.

The VLHC collaboration held a recent workshop on accelerator technologies at TJNAF in which many relevant instrumentation concepts were developed and discussed. The proceedings are available at http://www.vlhc.org/AT_proc.html. 


\section{Why Worry About VLHC Instrumentation?}

The main question to be addressed is cost. While a new hadron collider energy frontier will eventually be built, the time at which it will be built is largely a function how much progress is made in reducing the total project cost. If the machine is sited at Fermilab, only the costs of the final collider (plus detectors!) are incurred. The highlevel breakdown of the SSC accelerator and systems costs given by Marriner (http://www.vlhc.org/lakeg/Marriner.pdf) is worth study. Of the $\$ 2.7 \mathrm{~B}$ collider costs, about $75 \%$ are magnet and associated systems such as cryogenics, spool pieces, magnet power supplies, etc. Thus VLHC R\&D activities have appropriately concentrated on these areas.

Instrumentation and controls contributed less than $3 \%$ of the SSC final collider costs. The beam characteristics of the VLHC $(53 \mathrm{MHz}$ bunched beams, millimeter beam sizes, and $\sim 100 \mathrm{~mA}$ circulating currents) are similar to existing machines. Why then should we be concerned with VLHC instrumentation at all? The first reason is that the VLHC will inevitably be physically larger than predecessor machines and reliability and maintenance issues will become more critical. Instrumentation and controls impact reliability to a degree out of proportion to their fraction of project costs. Fortunately accelerators are not alone in their desire for high-reliability operation of remotely maintained systems, and proven industrial methodologies exist for analyzing and obtaining high reliability.

A second reason to study VLHC instrumentation is that different technical approaches to the VLHC vary significantly in their demands upon instrumentation. Instrumentation considerations could well be one of the deciding factors in the choice of magnet technologies for the VLHC. In addition, the development of advanced technologies (consider the advantages of robotic "search-and-secure" operations in a long tunnel!) can benefit the field as a whole.

A final reason to consider instrumentation at this point is that we believe that there are ways to make the magnets and cryogenics considerably cheaper than SSC/LHC. When that happens, the electronics costs will no longer be as small a fraction of the total.

\section{THREE APPROACHES TO THE VLHC.}

Three technical approaches are currently being pursued for the VLHC. These are characterized by the magnetic bend field strength and project structure. All are being pursued with an eye towards overall cost reduction of the VLHC.

\section{High Field VLHC}

This approach targets the production of 11-14T magnets with (1.3-1.5 times the strength of the LHC magnets) in order to reduce the machine circumference. New superconductors (modified $\mathrm{Nb} 3 \mathrm{Sn}, \mathrm{Nb3 \textrm {Al }}$ or high-temperature superconductor) should allow the magnets to operate at $4.5 \mathrm{~K}$ temperature of the SSC instead of the more-expensive $1.8 \mathrm{~K}$ operating temperature of the LHC. Various modifications of the 
2-D cross section are being considered to simplify coil winding and potentially reduce costs. Depending on the beam energy and bend field finally chosen the synchrotron damping time of the machine may be in the range of one to several hours. Significant technical challenges of this approach are that the superconductors in question are brittle and that the synchrotron radiation from $50 \mathrm{TeV}$ protons will require the beam screen to absorb many times higher power than the LHC beam screens.

\section{Low Field VLHC}

This approach recognizes that many of the magnet costs are associated with the cold bore vacuum system, high magnetic stored energy, and complex cryogenic systems of conventional superconducting magnets. Therefore this approach selects a 2-Tesla, 2-in-1 warm-iron design energized by a single-turn superconducting coil. The magnet consists essentially of a "double-C" iron yoke with two beam gaps placed around a 100kA superconducting transmission line. The iron pole tips produce a modest $(5 \% / \mathrm{cm})$ alternating-gradient field which eliminates the need for quadrupoles. Thus the magnets can be up to several cells long, and lengths as long as $250 \mathrm{~m}$ are being considered.

\section{Staged Approach to the VLHC}

In this hybrid approach, ultra-high energies are obtained in a series of more affordable stages. The first stage, inexpensive low-field magnets are placed in a tunnel about 20 miles in radius to provide a collision energy of $40 \mathrm{TeV}$, about 3 times that of the LHC. This stage establishes the tunnel, detectors, and a minimal set of magnets, power supplies, and cryogenics for that tunnel. The plan then envisions placing a series of higher strength magnets into the tunnel to increase the collision energy. "Medium field" magnets (similar to RHIC magnets) would allow a collision energy of $100 \mathrm{TeV}$ in this tunnel. Ultimately, 10-13 Tesla magnets could provide collision energies of 200-250 TeV. At each stage in this plan, a unique and stable physics program is maintained, and progression to the next phase can take place as technological and financial progress allow. This entire scope of this project could justifiably be termed the "Proton Microscope" since its ultimate goal is to explore the universe at the scale of one micro-Fermi - one-millionth the size of the proton.

\section{INSTRUMENTATION FOR HIGH-FIELD (COLD IRON) MAGNETS}

From the point of view of instrumentation and control, a machine based on medium or high-field cold-bore magnets will look similar to SSC or the LHC. Signals from BPM's, vacuum gauges, quench voltage taps, and so on emerge from cryogenic connections through the insulating vacuum. As in the LHC, the high-field magnets will probably require continuous racks of electronics underneath the dipoles around the circumference of the machine. Most of this equipment will be associated with the quench detection and protection circuitry, which will be at least as critical for high- 
field magnets as for the LHC. For economic reasons, these electronics will be located in the tunnel and their radiation resistance will be important. The LHC is planning to put an unprecedentedly large amount of electronics in the tunnel, and their experience in this regard will be very valuable. The LHC also contains complicated cryogenic interconnects between the 2-in-1 magnet cold mass and the cryogenic service piping. These interconnects occur at quad locations and require significant cryogenic instrumentation and valve control electronics.

Due to the high inductance and stored energy of the high field magnets, the ramping power supplies and energy dump switches must be located around the ring circumference. As in the Tevatron and LHC, these power supplies must be accurately synchronized during ramps and well regulated during stores.

\section{INSTRUMENTATION FOR LOW-FIELD (WARM IRON) MAGNETS}

From the point of view of beam instrumentation, the warm-iron magnets look like a conventional magnet with an extruded aluminum vacuum system. Since the machine is essentially straight, it is planned to directly weld the aluminum beam pipe sections together (no bellows) and mechanically connect them to the magnet iron to prevent bucking during bake out. BPM's will be conventional. Loss monitors (see below) are long cable-style BLM's which span the distance between arcs and are read out at both ends.

Due to the low inductance and magnetic stored energy, there is only one dipole power supply for the whole ring. There is only one quench detection circuit, which is located right at the power supply terminals. Magnet energy dump switches are located at $\sim 20 \mathrm{~km}$ intervals and are specialized sections of transmission which are deliberately quenched and rapidly re-cooled following a quench. The cryogenic system is straight piping, with cool-down and crossover valves every $\sim 8 \mathrm{~km}$. The exact location at which a quench occurred is identified by time-of-flight of the shock wave pressure pulse down the transmission line to the nearby pressure transducers (which can be kilometers away). Thus the tunnel will be completely bereft of instrumentation in the long distance $(250 \mathrm{~m})$ between quad locations.

\section{Beam Loss Monitor Systems}

High field magnets such as the LHC need large iron yokes to constrain the superconducting coils. These iron yokes help with radiation shielding of the in-tunnel electronics, but make it difficult to monitor losses anywhere except the spool pieces where the beam pipe is relatively unshielded. Thus the LHC (and presumable the high field VLHC) will rely on point-like loss monitors near the quad locations. A significant tradeoff regards whether the BLM's are located near the beam pipes (and therefore inside the insulating vacuum) or far from the beam pipes (where they will have difficulty resolving which of the two cold bores the losses are coming from). Finally, the high-field magnets themselves are some of the most sensitive loss 
monitors, since $\sim 100 \%$ of the energy from beam losses goes into the cold mass and they will immediately quench if significant losses occur at $\mathrm{TeV}$ energies.

The low field magnet differs in two respects. Firstly, it has much less shielding for external devices because it is a C-magnet and the overall iron cross section is smaller. Secondly, most of the energy from beam losses is absorbed in the room-temperature parts and only about $2 \%$ of the energy makes it into the cryogenic transmission line. The transmission line itself is a cable-in-conduit conductor with the NbTi strand braided and soaked in liquid helium, which gives it a substantial enthalpy margin compared to normal magnets. The overall effect is that it is much less sensitive to beam induced quenches. Operationally this is very pleasant feature, but it leaves open the possibility that chronic losses somewhere between quad locations could go undetected. For this reason, a continuous "cable style" beam loss monitor is desirable.

\section{Permanently Sealed Coaxial BLM's}

Existing "cable style" BLM's are ionization chambers built from with Heliax or similar coaxial cables. These typically flow gas (or periodically change the gas) in the annular space between conductors to avoid gain drop due to electron-absorbing impurities in the gas. Such impurities are to be expected from plastic, especially in a radiation field. On the other hand, the "Tevatron Style" BLM's represent an existence proof that permanently sealed metal and glass loss ionization loss monitors can work for decades without maintenance. To get the best of both worlds, we are embarking on an R\&D program to develop a permanently sealed cable-style BLM. Only metal, glass, and ceramic components will be used. The outer jacket will be a 1/2" soft Aluminum tube purchased in $2 \mathrm{ft}$ diam $\mathrm{x}$ 10ft long coils. The tube was extruded with an argon purge and hopefully will not need special cleaning. The inner conductor will be a copper wire. The first attempt at a coaxial spacer will be a fiberglass rope helically wrapped around the inner conductor. The glass rope has been baked out at high temperature during manufacture and should not need cleaning. If this fails, glass or ceramic beads will be used to support the center conductor. A sufficient quantity of these may be obtained in exchange for one of several inconsequential islands near the mouth of the Hudson River. The end connections will be permanently sealed and brazed to coaxial ceramic feed-thrus. We expect the final product to be easier to bend than Heliax but electrically inferior.

For low field VLHC, the bend radius is not important since the BLM cable will be permanently installed in the factory and runs the entire length of the magnet. Two BLM's are planned to separate losses from the two bores of each magnet. They will be read out (and potentially powered) from each end for redundancy. If readout includes high-bandwidth timing information, this can be used to localize losses within each cell. 


\section{Beam Abort Logic (Low Field VLHC)}

The stored energy in the beams of the VLHC is of order $1 \mathrm{GJ}$, which is enough to fry a few hot dogs or melt several cubic feet of stainless steel. So it would be desirable if the beam abort were reliable.

One aspect of this is to have an extremely reliable loss monitor system. The loss monitor system must be reliable in both directions. Firstly, it must not miss any losses which occur. Secondly, must also be reliable in the sense of not bringing the machine down if individual channels are broken or noisy, or even if the electronics module at one quad location loses power or goes incommunicado. The cable-style BLM, with redundant readout on opposite ends of the cable by separate electronics modules at different quad locations, meets that requirement.

A second aspect is that the beam abort must be an all-or-nothing proposition, i.e. no partial aborts or other kicker misfires can be tolerated. The basic strategy is to have multiple kicker modules, with sufficient total strength that e.g. any 4 out of 6 modules have enough kick to generate a tolerably clean abort. This provides some immunity from module failure. In addition, backup logic that automatically fires all modules when any module fires for any reason will prevent partial aborts with high reliability. This type of system was defined by the SSC and work continues along these lines for the LHC.

Beam aborts due to magnet quenches are not emergencies on an electronic time scale. The resistive voltage developed during a quench have initial voltages in the range of $1-10 \mathrm{~V}$, whereas the total flux in the magnet chain is 100,000 Volt-seconds for a $100 \mathrm{TeV}$ low-field machine. Thus there are tens of milliseconds available for the abort to react before the beam falls out of the machine due to a quench.

\section{Packaging of the In-Tunnel Electronics}

As has been previously remarked, the electronics layout of the High Field machines is likely to look similar to the SSC/LHC. In the case of the low field machine, there are several differences:

1) The magnets are long and the entire cable plant is installed and checked out at the factory. The magnets have an "extension cord" to a replaceable electronics module at each quad location.

2) The power dissipation is low, $1 \mathrm{~kW}$ per quad location or less. It is dominated by the warm corrector magnets.

3) The overall system power dissipation is low enough that no LCW system is required in the tunnel.

These three factors lead to the resurrection of the old idea of having the electronics in a small shielded "hole-in-the-wall" and dispensing with traditional crates, racks, instrumentation alcoves, etc. The quantity of identical instrumentation modules is sufficient to warrant a lot of engineering to make them small, low power, and robust. Heat pipes or convective "bubble pumps" could transport the heat out of the "hole-inthe-wall" to a heat sink which exchanges with the air flow in the tunnel. 


\section{Distributed Damping Systems for the Coupled-Bunch (RW) Instabilities}

VLHC work has contributed at least one interesting new concept to beam instrumentation. Because of its large circumference the low field VLHC has (at injection energy in a high-luminosity scenario) a growth time for the resistive-wall instability that is comparable to the revolution frequency. A solution to this difficulty (due to Marriner) is to simply distribute the damping system around the ring. A series of pickup/kicker pairs is distributed around the ring. Beam position errors sensed at the pickup are removed by kickers located 90 degrees in Betatron phase downstream. No single turn delay or digital memory is required since the pickup/kicker pair is simply canceling the RW wake field of a large number of preceding bunches. Foamfilled cables and audio-bandwidth amplifier are sufficient. By distributing of order 10 such systems around the circumference of the ring, the RW growth rate can be reduced to the point where it can be easily "cleaned up" by a conventional turn-by-turn damper system. There are no noise issues, since the RW damper circuit is only needed in ultra-high luminosity scenarios where the beam current and pickup signals are large. The distributed damper system is turned off after the beam is accelerated and the RW instability fades.

\section{Distribution Topologies: Network Fiber and AC Power}

In conducting preliminary work on $3 \mathrm{TeV}$ injector design study, we were confronted with a nearly identical set of choices for two seemingly dissimilar problems: AC power distribution and digital network fiber topology. The basic goal in either one is to be able to kill one or several feeders or substations (or optical links and network hubs) and have the system survive. One is immediately led to variants on the tree or ring topologies.

The optimal AC distribution topology from our point of view is a dual ring feeder system. In this system a $13.8 \mathrm{kV}$ feeder loops around the ring and stops at numerous substation transformers along the way. Cable capacity is sufficient that the ring can be fed entirely from either the clockwise or counterclockwise direction. Thus, remotecontrolled disconnects can allow the isolation of any failed feeder segment while still maintaining power to all substations.

The second tier (480VAC) power distribution takes place along a second parallel loop which connects to each instrumentation alcove as well as to the less frequent substations. Again, the cable capacity is sufficient that alcoves can be powered from the substation on either side of them. Remotely controlled disconnects allow the isolation of any $480 \mathrm{~V}$ cable segment without losing power to any alcove. Finally, the overall accelerator design is such that it can be safely operated with isolated quad locations without powered or dead.

At first glance the network fiber problem seems identical, with the substitutions Substations $\rightarrow$ Network Hubs and Power Feeders $\rightarrow$ Fiber Links. However the economics of optical fibers turn out to be completely different than those of copper power lines. It costs very little to double the number of fibers in an optical fiber bundle that you are installing, whereas doubling the ampacity of a large feeder very 
nearly doubles its cost. Thus our conclusion is to adopt a much more tree-like structure for fiber optic distribution, with large numbers of independent fibers fanning out to individual destinations from a common control point. A nearest-neighbor link from quad-to-quad in the tunnel would serve as a backup in case of a single-fiber failure.

\section{ACKNOWLEDGMENTS}

The author thanks the many participants of the VLHC Accelerator Technologies workshop (as well as those of BIW'00) for many stimulating and occasionally fruitful discussions. 